\title{
Molecular Dynamics Simulation on Designed Antibodies of HIV-1 Capsid Protein (p24)
}

\author{
Hana Atiqah Abdul Karim ${ }^{1}$, Chatchai Tayapiwatana ${ }^{2}$, Piyarat Nimmanpipug ${ }^{3}$, Sharifuddin M. Zain ${ }^{1}$, Noorsaadah \\ Abdul Rahman ${ }^{1}$, Vannajan Sanghiran Lee ${ }^{1,3^{*}}$ \\ ${ }^{I}$ Drug Design and Development Research Group, Department of Chemistry, Faculty of Science, University of Malaya 50603 Lembah Pantai, \\ Kuala Lumpur Malaysia. \\ ${ }^{2}$ Division of Clinical Immunology, Department of Medical Technology / Biomedical Technology Research Unit, National Center for Genetic \\ Engineering and Biotechnology, National Science and Technology Development Agency, Faculty of Associated Medical Sciences, Chiang \\ Mai University, Chiang Mai 50200,Thailand. \\ ${ }^{3}$ Computational Simulation Modelling Laboratory (CSML), Department of Chemistry and Center of Excellence for Innovation in Chemistry \\ and Materials Science Research Center, Faculty of Science, Chiang Mai University, Chiang Mai 50200, Thailand.
}

Received: 10 October 2014 / Accepted: 30 November 2014

\begin{abstract}
:
Computational approaches have been used by the molecular biologists all around the world as a vital tool in developing and improving the functional and binding properties of proteins, particularly antibody-antigen ( $\mathrm{Ab}-\mathrm{Ag}$ ) complexes. Based on a previous work, it has been identified that several residues located in the complementary determining region (CDR) of antibody fragment of FAB23.5 may serve as a potential point mutation modification in order to improve the binding interaction and the inhibitory activity between the single chain variable fragment ( $\mathrm{scFv}$ ) antibody and the epitope variant of HIV-1 p24 capsid protein. In this study, several binding residues from the variable chain domain of scFv anti-p24 were modified. The designed antibody along with the bound antigen was then subjected for molecular dynamics (MD) simulation by using PMEMD.CUDA under Amber 12 with NVIDIA ${ }^{\circledR}$ Kepler $^{\circledR}$ GPUs system. GPU system has been fully utilized in this work as a mean to speed up the calculation time. From the simulated model, the newly possessed binding interaction of the modified AbAg complex were acquired by using the Molecular Mechanics/Poisson-Boltzmann surface area (MM-PBSA) and Molecular Mechanics/Generalized Born surface area (MM-GBSA), respectively.
\end{abstract}

Key words: Antibody, complementary determining region, hiv, molecular dynamics simulation, mm-gbsa, single chain variable fragment, single point mutation, molecular docking

\section{Introduction}

For the past three decades, Human Immunodeficiency Virus Type-1 (HIV-1) continues to come into sight as a global pandemic where it has claimed more than 25 million lives. And in recent times, it has been reported in year 2012 by World Health Organization (WHO) that they were approximately 35.3 million people that have been living with HIV viruses worldwide [1]. This resembles the huge numbers of the newly HIV-infected patients which is rather alarming. Principally, HIV is composed of two copies of positive single stranded RNA that codes for the virus's nine genes along with the presence of crucial elements such as viral protein p24, nucleocapsid protein $\mathrm{p} 7$, enzymes needed for the development of the virion, matrix protein $\mathrm{p} 17$, several types of viral proteins as well as proteins from the last host cell it infected. The viral capsid protein of p24 basically serves as the viral core of the HIV-1 virus where it contains the single-stranded RNA genome and is being abundantly present in the virus particle. Despite the structural role of the protein in forming the core of the mature virion, the capsid protein of p24 is also essential during the process of viral assembly as it plays a pivotal role in viral penetration or uncoating or both, a function which maybe mediated by binding p24 with human cellular proline rotamase cyclophilin A [2].

Antibodies have been under evaluation for their potential function in interacting with p24 and thus trigger the inhibitory activity. Of the antibodies, single chain variable fragment $(\mathrm{scFv})$ has been widely used for expressing antibody in intracellular way due to the smaller size and specificity possessed [3]. Interactions involved within the antibody-antigen complex are one of the described approaches in anti-HIV studies. Antibodyantigen complex of scFv-anti-p24 was studied and it suggested that anti-p24 scFv could aid in the treatment of HIV infections. Thus, in order to be acquainted on how antibody and antigen interacts with each other as well as to have the molecular basis on how to optimize the antibodies to passive immunization or even designing new drugs that mimicking the antibodies, the structural rules governing the $\mathrm{Ab}-\mathrm{Ag}$ interactions need to be understood beforehand [4].

\footnotetext{
*Corresponding author: Vannajan Sanghiran Lee,

E-mail: vannajan@gmail.com
} 
By implementing several computational approaches namely, molecular docking and molecular dynamics (MD) simulation to the scFv-anti-p24 complex, theoretical prediction on the most favorable binding interaction between the antibody and antigen can be done. From here, several useful insights can be obtained via the evaluation of the interaction energy, binding free energy as well as the pairwise decomposition or residuebased energy calculation of complexes in solution by using the molecular mechanics/poisson-boltzmann surface area (MM-PBSA) and molecular mechanics/ generalized born surface area (MM-GBSA) method [5]. All of these will then lead us into the possible mutation residues of the antibody thus improving the binding interaction and inhibitory activity of it. Or even better, by taking it into another level, this surely will guide us on designing the novel antibody that has a better binding interaction and inhibitory mechanism with the HIV-1 p24 epitope variant.

The use of GPUs will be chosen for this study because previous testing study revealed that use of a GPU was faster than using locally available CPUs (simulations using $1 \mathrm{GPU}$ were approximately $50 \%$ faster than those using 32 Xeon $3.4 \mathrm{GHz}$ CPU cores). Several previous studies have investigated the use of GPUs to accelerate the MD simulations [6-12]. In addition to that, GPUs provide promising systems for energy efficient scientific computing. It was found that use of a GPU consumed significantly less energy $(\approx 3 \mathrm{MJ}$ per nanosecond of dynamics using 1 GPU compared to 10 MJ per nanosecond of using 32 CPU cores). In this study, we aim to accelerate the computational research and engineering applications on molecular dynamics simulation with NVIDIA ${ }^{\circledR}$ Kepler $^{\circledR}$ GPUs for the large time scale simulation on the specific design novel antibody. The expected outcome is to investigate the behavior of this theoretically-generated antibody-antigen complex under the large time scale simulation with graphics processing units GPU.

\section{Experimental}

Molecular Dynamics (MD) simulation on the x-ray crystal structure

In order to determine the hot spot of the antibody complex, the X-ray crystal structure of it; HIV-1 p24 bound with the antibody (PDB ID 1AFV) was subjected to both energy minimization and molecular dynamics simulation by using PMEMD.CUDA from AMBER12 on GPUs Quadro 2000D manufactured by NVIDIA. The use of GPUs provides faster large time scale molecular dynamics simulation in order to obtain the trajectory files required from each simulation. Preparation of the complex was conducted by using ff12SB force field to describe the molecular characteristics of the complex followed with the solvation in a cubic box of TIP3P waters extending at least $10 \AA$ in each direction from the solute together with the addition of $\mathrm{Na}^{+}$ions which act as neutralizing counter ions. The cut-off distance was kept at $12 \AA$ to enumerate the nonbonded interactions. Initially, the temperature of each system was increased gradually from 0 to $310 \mathrm{~K}$ over a period of $60 \mathrm{ps}$. This was followed by 300 ps of NPT equilibration at 310.15 $\mathrm{K}$ and $1 \mathrm{~atm}$ pressure. Eventually, an unrestrained MD simulation was accomplished at a constant temperature of $310 \mathrm{~K}$ for a total simulation of $10 \mathrm{~ns}$. From there 500 snapshots were collected from both 3 ns and 9 ns trajectories in order to compare the initial phase and the final phase of the MD simulations respectively. Evaluation of the structural properties, root-meansquared-deviations (RMSD) of the backbone atoms, binding free energy analysis as well as the decomposition energy of the key residues were conducted and further analyzed which lead to the determination of the important key residues of the antibody. Calculation on the complex's energy was done by using both the Molecular Mechanics/PoissonBoltzmann surface area (MM-PBSA) and Molecular Mechanics/Generalized Born surface area (MM-GBSA) method.

\section{Single point mutation and molecular docking}

Auto-Mutate program that was developed in our own lab has been used to perform the single point mutation on the antibody. This program has been generated based on one of the script file obtained from MODELLER; namely Mutate Model and AutoDock Vina; an open source program to conduct molecular docking all in one program. The script provided by MODELLER allows the user to mutate and at the same time, optimize a single residue. First, the script takes a given PDB file and mutates the specified single residue selected by the user before the program optimized the particular residues' position. The conformation of the mutant is optimized by conjugate gradient whilst at the same time being refined by using some MD. Configuration files needed to run AutoDock Vina specific to each engendered mutants were generated where the grid box was being defined with such information: (i) number of points in $x, y$ and $z$ dimension are 39,23 , and 22 respectively, (ii) center grid box for $\mathrm{x}, \mathrm{y}$ and $\mathrm{z}$ center are 35.752, 20.693, and 95.448 respectively, and (iii) the spacing used was $1.0 \AA$.

\section{Screening on the interaction energy of the designed antibodies}

Basic screening on all the designed antibodies was conducted with the use of Discovery Studio 2.5 by comparing the interaction energy of the mutants only at the complementarity determining region (CDR); (VL: 31-36, 96-98) and (VH: 50, 93-105) of the antibody. From there, only the best design specific for each position were selected to undergo MD simulations.

\section{Molecular dynamics (MD) simulation on the designed antibodies}

The same procedure was applied to the $\mathrm{x}$-ray crystal structure of the HIV-1 p24 bound to all the three modified antibodies, I185K, E248K and G470R. 


\section{Results and Discussion}

The x-ray crystal structure of the wild type complex was subjected to Molecular Dynamics simulation (MDS) for $10 \mathrm{~ns}$. The root-mean-squared-deviations (RMSD) of the backbone atoms indicates that the complex was stable after $2 \mathrm{~ns}$, post-analysis was done by binding free energy and decomposed energy lead to the determination of the important key residues of the antibody. The decomposed energy of the residues at 2 ns and 9 ns trajectories is shown in Figure 1 where the key residues Ile185, Glu248, Gly470 have been indicated.

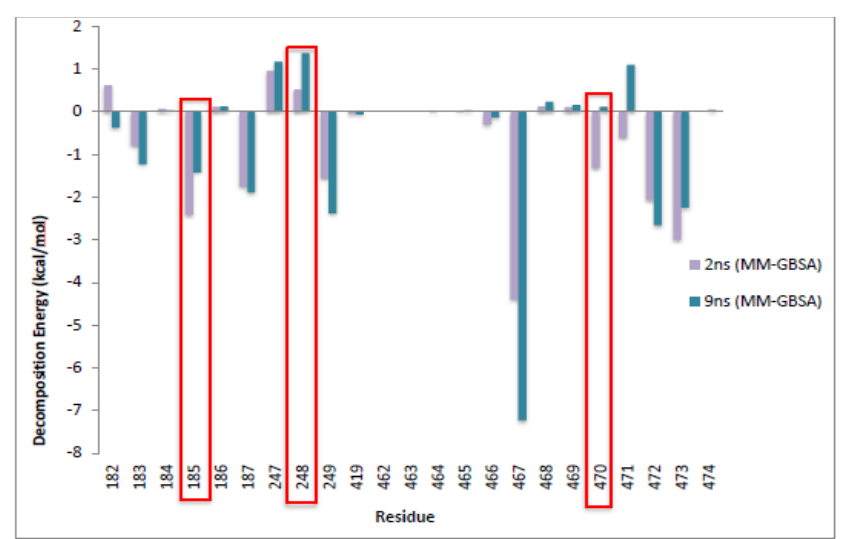

Figure 1. Decomposed energy of the residues of the wild-type complex.

Single point mutation of the key residues was performed by Auto-mutated script. All the above three residues were subjected for mutation by rest of the available amino acid residues one by one and were screened for interaction energies. The lowest interaction energy completed structures were obtained and investigate in the next section.

All the designed antibodies were screened for interaction energy using Discovery studio program and interaction energies of the residues were analyzed to obtain the best design. After analysis of the interaction energies residues (Lysine for residue Ile185 and Glu248 and Arginine for residue Gly470) were identified in Figure 2.

Binding free energies of the designed antibodies I185K, E248K and G470R were studied using both algorithms (MM-PBSA/GBSA) and it was found that the MMGBSA gave more promising results. It correlates with many of the protein-protein system where MM-GBSA protocol has been believed to achieve encouraging performance in reproducing the relative binding free energy. It was found that all the three designed antibodies produced good results as shown in Figure 2 and amongst those G470R was found to be the best one having the lowest energy.

The comparison of decomposed energies of the wildtype and the designed antibodies suggested that all the three designed antibodies have lower decomposition energies as compared to the wild-type suggesting a good binding of antigen and antibody. Figure 3 shows lower energy of designed antibody (G470R) as compared to that of wild type.

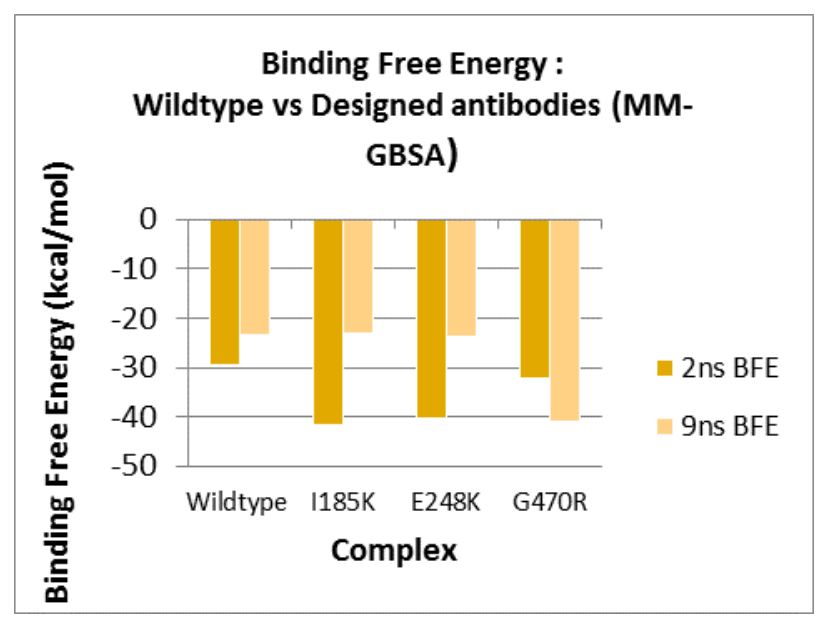

Figure 2. Binding Free energy of the residues of wild type and designed antibody using MM-GBSA method.

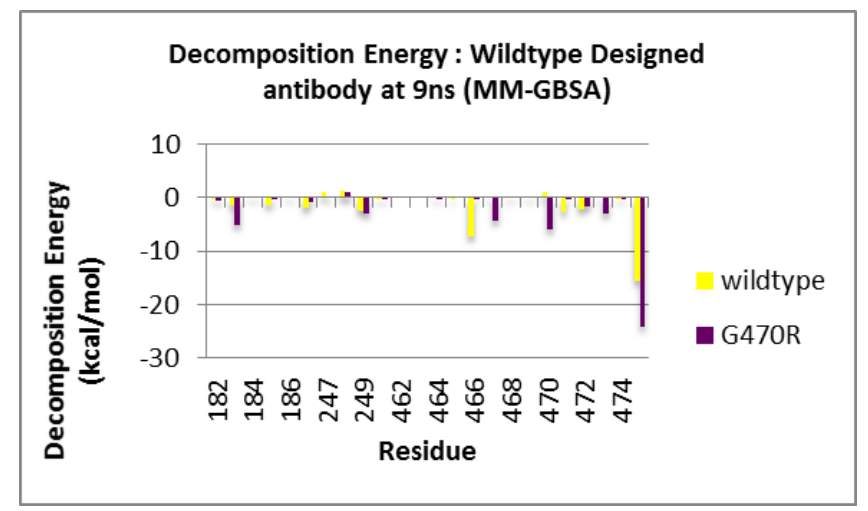

Figure 3. Decomposition energy of the residues of wild type and designed antibody (G470R).

\section{Conclusions}

Several key residues were identified for the modification of a novel antibody of HIV-1 p-24: VL 185, VL 248 \& VH 470. After mutation studies and interaction energy studies it was suggested that modification with lysine is best for Ile185, Glu248 and with Arginine for Gly470. Our best designed antibody of the Ab-Ag complex, G470R has shown to improve the binding interaction by having a low relative binding free energy (BFE) of 17.69 $\mathrm{kcal} / \mathrm{mol}$ and a low total decomposition energy ( $9 \mathrm{~ns})$ at the $\mathrm{CDR}$ region $=-24.17 \mathrm{kcal} / \mathrm{mol}$ (wild type -15.6 $\mathrm{kcal} / \mathrm{mol}$ ). This suggests that the designed complex is stable. Putting all together, the key residues in the AbAg complex of HIV-1 p-24 were identified and investigation of the behavior of the novel design antibody after modification to G470R suggests it to be a promising designed antibody. Further experimental testing is under investigated. 


\section{Acknowledgement}

This research is supported by University Malaya Research Grant under Computation and Informatics $(\mathrm{C}+\mathrm{i})$ Research Cluster/High Performance Scientific Computing Program (UMRG Project no. RP001C13ICT), UMBIO Research Cluster (RP002-2012D), Fundamental Research Grant Scheme (FP013-2013A). The CRYSTAL, University Malaya for Software Usage (Discovery Studio).

\section{References}

[1] Global Health Observatory (GHO). World Health Organization (WHO), retrieved from http://www.who.int/ gho/hiv/epidemic_status/cases_all_text/en.

[2] B.L. Kazemier, M.J.M. Koolen, L.J. Nijholt, R.O.B.H. Meloen, B.O.B.V.A.N. Gemen, H.R. Hoogenboom, and J. Arends, Selection of recombinant, library- derived antibody fragments against p24 for human immunodeficiency virus type 1, Diagnostics, 5(5), 1998, 636-644.

[3] V.S. Lee, P. Tue-ngeun, S. Nangola, K. Kitidee, J. Jitonnom, P. Nimamanpipug, S. Jiranusomkul, and C. Tayapiwatana, Journal of Molecular Immunology, 9, 2010, 82-990.

[4] M. Pedotti, L. Simonelli, E. Livoti, and L. Varani, International Journal of Molecular Sciences, 12(1), 2011, 226-251.
[5] P. Tue-ngeun, P. Nimmanpipug, N. Lawan, S. Nangola, C. Tayapiwatana, and V.S. Lee, Bio. Med. Research International, 2013, 713585 -713597.

[6] J.C. Phillips, J.E. Stone, and K. Schulten, Adapting a message driven parallel application to GPU-accelerated clusters. SC '08: Proceedings of the 2008 ACM/IEEE Conference on Supercomputing; IEEE Press: Piscataway, NJ, 2008, 1-9.

[7] M.S. Friedrichs, P. Eastman, V. Vaidyanathan, M. Houston, S. Legrand, A.L. Beberg, D.L. Ensign, C.M. Bruns, and V.S. Pande, J. Comput. Chem., 30, 2009, 864-872.

[8] P. Eastman and V.S. Pande, J. Comput. Chem., 31, 2010, 1268-1272.

[9] M.J. Harvey, G. Giupponi, and G.D. Fabritiis, J. Chem. Theory Comput., 5, 2009, 1632-1639.

[10] M.J. Harvey and G.D. Fabritiis, J. Chem. Theory Comput., 5, 2009, 2371-2377.

[11] S.S. Hampton, P.K. Agarwal, S.R. Alam, and P.S. Crozier, Towards microsecond biological molecular dynamics simulations on hybrid processors, Proceedings of the International Conference on High Performance Computing and Simulation, June 28-July, 2, 2010, 98-107, doi: 10.1109/HPCS.2010.5547149.

[12] W.M. Brown, P. Wang, S.J. Plimpton, and A.N. Tharrington, Comput. Phys. Commun., 182, 2011, 898-911. 